\title{
RESONANCE VIBRATION OF RAILWAY BRIDGES SUBJECTED TO PASSING VEHICLES
}

This paper discusses some issues related to dynamic effects in railway bridges focussed on the simulation of the resonant vibration for the small and medium span simply supported railway bridges subjected to a series of moving vehicle. Presented parametric study is concerned with the dynamic deflection of the simply supported railway truss bridge of the span $L_{b}=38 \mathrm{~m}$, due to the series of the ten moving loads representing a conventional train with the IC-coaches employed in Slovakia, with the impact to the resonance speed $c_{2 \text { res }}=65.03 \mathrm{~m} / \mathrm{s}=234.11 \mathrm{~km} / \mathrm{h}$. The deflection amplitude ${ }^{\left(c_{2}=33\right)} w_{(1),\left(P_{1}, P_{2} \ldots P_{N}\right)}\left(L_{b} / 2, t\right)$ increase with an increasing number of load forces $n=1,2, \ldots N$ forces moving along the bridge.

Keywords: The dynamic response, railway bridge deflections, resonance amplitudes, the modal superposition method.

\section{Introduction}

The dynamic response of railway bridges, subjected to moving trains is influenced by a number of factors such as the speed of load, the bridge span, natural frequencies of the bridge and railways vehicles, the inertia and damping of the two interaction systems (vehicles and the bridge), the distance between the vehicles, and arranging axles of vehicles. At present, the actual question for the bridge loading follows from high speed trains, which may consist of a number of identical cars connected together moving with the speed $c$. In those cases, the resonance caused by configuration of the train, consisting of a number of vehicles of similar types (Fig. 1), may occur, especially at high speed ranges.

To solve indicated problems one needs to apply the special dynamic analysis depending on the type of the bridge structure with regards to the static determination of the structure. For statically indeterminate structures, like continuous deck bridges or frame structures, more sophisticated methods of analysis (FEM) must be applied. For the simple bridges, the solution is based on the modal superposition method [1-3].

Railway vehicles travelling along the bridge are modelled as a series of identical moving loads and assuming that the vehicle/ bridge mass ratio is small $m_{v}<\mathrm{m}_{\mathrm{b}}$ and loads move along the bridge, the close form of solution can be obtained. The dynamic displacement $w(x, t)$ and acceleration $\ddot{w}(x, t)$ of the bridge are governed at different extents by the two sets of frequencies:

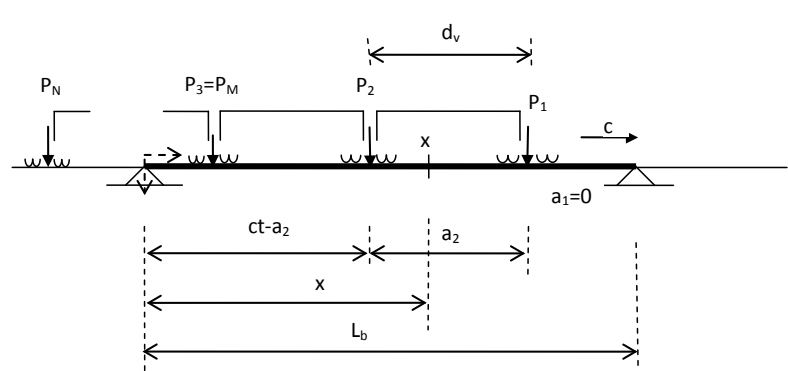

Fig. 1 Loading of the bridge by a series of identical IC-vehicles

- the driving frequency of a vehicle crossing the bridge, $\omega_{(j), d r}$ $j=1,2 \ldots$,

- the natural frequency of the bridge $\omega_{(j)}, j=1,2, \ldots$

One of the actual problems is the solution of the dynamic behaviour of the bridge subjected to a series of identical loads $\sum p_{n}, n=1,2, . . N$, with identical space intervals $d_{v}$ (the length of the vehicle) passing the bridge with a constant speed $c[4]$ as is shown in Fig. 2.

In this paper the dynamic behaviour of the simply supported railway bridge with the span $L_{b}=38 \mathrm{~m}$, subjected to the successive identical moving loads is solved. The close form solution is obtained by means of the modal superposition method [1-2, 5-6]. The presented parametric study is focused on the dynamic deflection of the bridge at the mid-span ${ }^{(c)} w\left(L_{2} / 2, \mathrm{t}\right)$ due to loading of the conventional train with the Slovak IC-coaches for the resonance speed $c_{2 \text { res }}=65.03 \mathrm{~m} / \mathrm{s}=234.11 \mathrm{~km} / \mathrm{h},[7]$.

\footnotetext{
* Milan Moravcik, Martin Moravcik

Faculty of Civil Engineering, University of Zilina, Slovakia

E-mail: mimo@fstav.uniza.sk
} 
Using the analytical approach, the key parameters that govern the dynamic displacement response - the vertical beam vibration ${ }^{(c)} w_{\left(P_{1}, P_{2} \ldots P_{N}\right)}(x, t)$, for $M$ loads moving on the railway steel girder of the length $L_{b}=38 \mathrm{~m}$ loaded by the ten IC-cars length $d_{v}=24.5 \mathrm{~m}$ with the magnitude $P_{v}=524 \mathrm{kN}$, Fig. 1 .

\section{Formulation of the theory for the bridge response induced by moving load series}

Consider a simply supported beam (without damping) subjected to a series of concentrated constant loads $P$, which are moving at a uniform speed $c$, in the meaning of Fig. 2. The motion equation for the beam subjected to periodical loading of moving load series can be writing as

$E I \frac{\partial^{4} w(x, t)}{\partial x^{4}}+m_{1} \frac{\partial^{2} w(x, t)}{\partial t^{4}}=\sum_{n=0}^{M-1} P \delta\left(x-c\left(t-\frac{n d_{v}}{c}\right)\right)$,

$n=1,2, \ldots M$,

where:

$w(x, t)$ - is the displacement of the beam at point $x$ and time $t[\mathrm{~m}]$, $E I$ - is the bending stiffness of the beam $\left[\mathrm{kNm}^{2}\right]$,

$m_{1}$ - is the mass per unit length of the beam [t],

$P_{v, i} \equiv P_{i}$ - is the loading force [kN],

$\delta(x)$ - is the Dirac function,

$c$ - is a uniform speed of moving loads $[\mathrm{m} / \mathrm{s}]$,

$L_{b}$ - is the span length of the bridge [m],

$d_{v}$ - is the identical interval between loading forces [m].

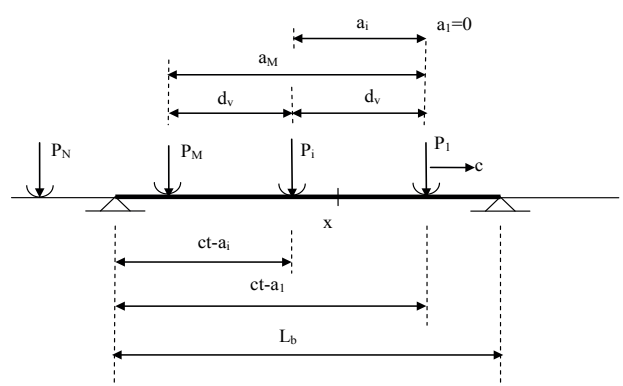

Fig. 2 The single-span bridge subjected to moving loads $\sum_{n} P_{n}$

For a simple beam the solution of the vertical deflection $w(x, \mathrm{t})$ in Equation (1) becomes the harmonic analysis [1,2] The particular solution $w(x, \mathrm{t})$ for a simply supported beam can be expressed in terms of the modal time coordinates $q_{(j)}(t)$ for the beam vibration and the modal shapes $\phi_{j}(x)$ as

$w(x, t)=\sum_{j} q_{(j)}(t) \phi_{j}(x)=\sum_{j} q_{(j)}(t) \sin \left(\frac{j \pi x}{L_{b}}\right)$,

$j=1,2,3, \ldots$

where:

$q_{(j)}(\mathrm{t})$ - are the generalized coordinates that define the amplitude of vibration with time $t$, $\phi_{j}(x)=\sin \left(\frac{j \pi x}{L_{B}}\right)$ - is a fundamental mode shape and for the simply beam is of the sinusoidal type (the first mode is an one-half cycle; the second mode is a full cycle).

Thus, the dynamic deflection may be represented by the summation of modal components. When the first and last moving load on the bridge span be $P_{I}$ and $P_{M}$ at a time $t$, Equation (1) can be expressed in terms of the generalized coordinates as

$\frac{d^{2} q_{(j)}(t)}{d t^{2}}+\omega_{(j)}^{2} q_{(j)}(t)=\frac{2 P}{m_{1} L_{b}} \sum_{n=0}^{M-1} \sin \left(\frac{j \pi c}{L_{b}}\left(t-\frac{n d_{v}}{c}\right)\right) H_{n}$

$n=1,2, \ldots M$

for $M$ forces moving on the bridge,

where:

$H_{n}=\theta\left(t-\frac{n d_{v}}{c}\right)-\theta\left(t-\left(\frac{n d_{v}}{c}+\frac{L_{b}}{c}\right)\right)$ is the Heaviside function determining whether the load $P$ is on the bridge or not.

The modal coordinate $q_{(j)}(t)$ for the $j$-th mode of vibration of the beam from Equation (3) can be expressed as:

$$
\begin{aligned}
q_{(j)}(t)= & \frac{\hat{q}_{(\mathrm{j}) \mathrm{st}}}{1-\alpha_{(j)}^{2}} \sum_{n=0}^{M-1}\left[\sin \left(\omega_{(\mathrm{j}) \mathrm{dr}}\left(t-\frac{n d_{v}}{c}\right)\right)\right. \\
& \left.-\alpha_{(j)} \sin \left(\omega_{(j)}\left(t-\frac{n d_{v}}{c}\right)\right)\right] H_{n}(t)
\end{aligned}
$$

where:

$\hat{q}_{(j) s t}=\frac{2 P}{m_{1} L_{b} \omega_{(j)}^{2}}=\frac{2 P L_{b}^{3}}{j^{4} \pi^{4} E I}$ - is the modal amplitude - the static deflection caused by the force $P$ with respect to the $j$-th mode,

$\omega_{(j)}=\frac{\pi j^{2}}{L_{b}^{2}} \sqrt{\frac{E I}{m_{1}}}$ - is the $j$-th circular frequency of the beam vibration,

$\alpha_{(j)}=\frac{\omega_{(j) d r}}{\omega_{(j)}} \equiv \frac{c}{c_{c r}}$ is the non-dimensional speed parameter,

$c_{c r}=2 f_{(1)} L_{b}=\frac{\omega_{(1)} L_{b}}{\pi}$ is the critical speed,

$\omega_{(j) d r}=\frac{j \pi c}{L_{b}}-$ is the circular driving frequency of the moving force for the $j$-th mode of vibration.

\subsection{The single-mode analytical solution}

The vertical deflection $w(x, \mathrm{t})$ in Equation (1) for a simply supported beam for a moving load problem can be well simulated by considering the first mode of vibrations $\omega_{(1)}$ only. The corresponding modal coordinate from Equation (4), taking into account $M$-vehicles on the bridge, is given by superposition of a forced response (a quasi-static response) due to the moving load and a transient response (the dynamic part of the response), can be expressed as follows:

$$
\begin{aligned}
{ }^{(c)} q_{(1),\left(P_{1}, P_{2} \ldots P_{M}\right)}(t)= & \frac{\hat{q}_{(1) s t}}{1-\alpha_{(1)}^{2}} \sum_{n=0}^{M-1}\left[\sin \left(\omega_{(1) d r}\left(t-\frac{n d_{v}}{c}\right)\right)-\alpha_{(1)}\right. \\
& \left.\sin \left(\omega_{(j)}\left(t-\frac{n d_{v}}{c}\right)\right)\right] H_{n}(t)
\end{aligned}
$$


where:

$\alpha_{(1)}=\frac{\omega_{d r(1)}}{\omega_{(1)}}=\frac{\pi c}{L_{b} \omega_{(1)}}=\frac{c}{c_{c r}}$ is the non-dimensional speed parameter corresponding to the first mode of vibrations $\omega_{(j)}$.

The vertical deflection ${ }^{(c)} w_{(1),\left(P_{1}, P_{2}, \ldots, P_{N}\right)}\left(L_{b} / 2, t\right)$ for the mid-span $x=L_{b} / 2$ is equal to the modal coordinate ${ }^{(c)} q_{(1),\left(P_{1}, P_{2}, \ldots P_{N}\right)}\left(L_{b} / 2, t\right)$, because $\sin \left(\frac{\pi x}{L_{b}}\right)=\sin (\pi / 2)=1$.

${ }^{(c)} w_{(1),\left(P_{1}, P_{2}, \ldots P_{N}\right)}\left(L_{b} / 2, t\right)={ }^{(c)} q_{(1),\left(P_{1}, P_{2}, \ldots, P_{N}\right)}\left(L_{b} / 2, t\right)=$ $=\frac{\hat{q}_{(1) s t}}{1-\alpha_{(1)}^{2}}(1,0) \sum_{n=0}^{M-1}\left[\sin \left(\omega_{(1) d r}\left(t-\frac{n d_{v}}{c}\right)\right)-\right.$

$\left.-\alpha_{(1)} \sin \left(\omega_{(j)}\left(t-\frac{n d_{v}}{c}\right)\right)\right] H_{n}(t)$

\subsection{Displacement response at the mid-span of the beam}

The important practical significance is just the dynamic deflection ${ }^{(c)} \boldsymbol{W}_{(1),\left(P_{1}, P_{2} \ldots P_{N}\right)}\left(L_{b} / 2, t\right)$ at the mid-span $x=L_{b} / 2$ for loads of a moving series $\left(\mathrm{P}_{P}, \mathrm{P}_{2}, \ldots \mathrm{P}_{N}\right)$. If the number of the loads, moving out of the span, is $K$ and the number of moving forces moving just on the span is $M$ at the time $t$, the displacement response of the beam can be generalized considering the superposition of the next loading effects $[2,5-6]$ :

${ }^{(c)} \boldsymbol{w}_{(1),\left(P_{1}, P_{2}, \ldots P_{N}\right)}\left(L_{b} / 2, t\right)={ }^{(c)} q_{(1),\left(P_{1}, P_{2}, \ldots P_{N}\right)}\left(L_{b} / 2, t\right) \phi_{1}\left(L_{b} / 2\right)=$ $=\frac{\hat{w}_{(1) s t}\left(L_{b} / 2\right)}{1-{ }^{(c)} \alpha_{(1)}^{2}}(1,0) \sum_{n=K}^{M-1} \sin ^{(c)} \omega_{(1) d r}\left(t-\frac{n d_{v}}{c}\right)-$

$-\frac{\hat{w}_{(1) s t}\left(L_{b} / 2\right)}{1-{ }^{(c)} \alpha_{(1)}^{2}}(1,0) \sum_{n=K}^{M-1}{ }^{(c)} \alpha_{(1)} e^{-\omega_{d}\left(t-\frac{n d_{v}}{c}\right)} \sin \omega_{(1)}\left(t-\frac{n d_{v}}{c}\right)-$

$-\frac{\hat{w}_{(1) s t}\left(L_{b} / 2\right)}{1-{ }^{(c)} \alpha_{(1)}^{2}} \sum_{n=0}^{K-1}\left({ }^{(c)} \alpha_{(1)} e^{-\omega_{d}\left(t-\frac{n d_{v}}{c}\right)} \sin \omega_{(1)}\left(t-\frac{n d_{v}}{c}-\frac{L_{b}}{c}\right)\right)-$

$-\frac{\hat{w}_{(1) s t}\left(L_{b} / 2\right)}{1-{ }^{(c)} \alpha_{(1)}^{2}} \sum_{n=0}^{N-1}\left({ }^{(c)} \alpha_{(1)} e^{-\omega_{d}\left(t-\frac{n d_{v}}{c}-\frac{L_{b}}{c}\right)} \sin \omega_{(1)}\left(t-\frac{n d_{v}}{c}-\frac{L_{b}}{c}\right)\right)$

for $n=1,2,3, \ldots N=M+K$, and for $\sin (\pi / 2)=1$.

The first term in Equation (7) represents the component corresponding to each moving load $P$ travelling over the beam - it produces the force vibration response (the quasi-static part of the response). The second and the third term in Equation (7) produce the free vibration response (the dynamic part of the response) considering forces moving just on the beam and forces moving away of the span, respectively. The fourth term in Equation (7) represents the free vibrations' term after all the moving loads left the span. Thus, the beam response ${ }^{(c)} w_{(1),\left(P_{1}, P_{2}, \ldots P_{N}\right)}\left(L_{b} / 2, t\right)$ can be symbolically written as

${ }^{(c)} \boldsymbol{w}_{(1),\left(P_{1}, P_{2}, \ldots P_{N}\right)}\left(L_{b} / 2, t\right)={ }^{(c)} w_{(1) s t,\left(P_{1}, P_{2}, \ldots, P_{W}\right)}\left(L_{b} / 2, t\right)+$

$+{ }^{(c)} \boldsymbol{w}_{(1) d y y_{,}\left(P_{1}, P_{2}, \ldots, P_{H}\right)}^{(1)}\left(L_{b} / 2, t\right)+{ }^{(c)} \boldsymbol{w}_{(1) d y,\left(P_{1}, P_{2}, \ldots, P_{k}\right)}^{(2)}\left(L_{b} / 2, t\right)$

\subsection{Condition of the resonance}

When all the moving loads have passed the beam, the force part of the vibration terminates immediately. However, the free vibration part continues to exit until it is eventually damped out. Our parametric studies for different train speeds show that both the phenomena of resonance and cancellation are related to the free vibration component of the beam vibrations only. Thus, the sum of the free vibration response components by each moving load can result in the resonance if the total number $N$ of the moving loads is large enough.

If the forces act on the bridge at equal distances $d_{v}$ then their repeated action can cause a resonant vibration. The resonant condition follows from the well know condition [5-7]:

$k f_{\left(d_{v}\right)}=f_{(1)} \rightarrow k \frac{c}{d_{v}}=\frac{\omega_{(1)}}{2 \pi}, k=1,2,3 \ldots$,

The resonant condition (9) is calculated from the time necessary for crossing the distance $d_{v}$ at the speed $c$ which is equal to the $k$-multiple of the natural beam vibration $f_{(l)}$. From Equation (9) results the resonant speed under the condition $\cos \left(\omega_{(1)} L_{b} / c\right)[7]$ :

$c_{(i, r e s)}=\frac{\omega_{(1)} d_{v}}{2 \pi k}, k=1,2,3 \ldots$

Critical speeds $c_{(i, r e s)}$ according to Equation (10) are given in Table 1.

Critical speeds for train with IC-cars $\left(d_{v}=24.5 \mathrm{~m}\right)$

and for $L_{b}=38 \mathrm{~m}$

Table 1

\begin{tabular}{|c|c|c|}
\hline$k$ & $c_{i, r e s}=\frac{\omega_{(1)} d_{v}}{k 2 \pi}$ & for $L_{\mathrm{b}}=38 \mathrm{~m}, d_{v}=24.5 \mathrm{~m}$ \\
\hline 1 & $\mathrm{c}_{1, \mathrm{res}}=130.1 \mathrm{~m} / \mathrm{s}$ & $\cos \left(\omega_{(1)} L_{b} / c_{1}\right)=-0.9506$ \\
\hline 2 & $\mathrm{c}_{2, \mathrm{res}}=65.03 \mathrm{~m} / \mathrm{s}$ & $\cos \left(\omega_{(1)} L_{b} / c_{2}\right)=0.8072$ \\
\hline 3 & $\mathrm{c}_{3, \mathrm{res}}=43.35 \mathrm{~m} / \mathrm{s}$ & $\cos \left(\omega_{(1)} L_{b} / c_{3}\right)=-0.5843$ \\
\hline 4 & $\mathrm{c}_{4, \mathrm{res}}=32.52 \mathrm{~m} / \mathrm{s}$ & $\cos \left(\omega_{(1)} L_{b} / c_{4}\right)=0.3034$ \\
\hline
\end{tabular}

From the above resonance considerations follow:

- The dynamic response is strongly influenced by the freevibration components in Equation (7) if the total number of moving loads is large enough.

- The resonant-response amplitude decreases when the value of $k$ increases.

- The ratio $L_{b} / c$ of the span $L_{b}$ to the velocity of the moving loads $c$ expressively influences the response amplitude.

- The difference between the phase angles in the dynamic components in Equation (7) affects the phenomena of resonance and its cancellation.

- The increase of the damping is an effective measure to reduce resonant - amplitude. 
3. Dynamic deflection due to a series of moving load $\left(P_{1}+P_{2}+\ldots . .+P_{10}\right)$ crossing the beam

The steel truss bridge $L_{\mathrm{b}}=38 \mathrm{~m}$ is subjected by the series of 10 IC-cars $\left(P_{1}+P_{2}+\ldots+P_{10}\right)$ in the sense of Fig. 3. The load of equal weights $P=524 \mathrm{kN}$ is spaced at the car interval $d_{v}=24.5 \mathrm{~m}$, the train speed is $c_{2, \text { res }}=65.03 \mathrm{~m} / \mathrm{s}$, see Table 1 . Evaluation of all time dynamic response waveforms were applied from a programming system Wolfram Mathematica 8.0.1[8].

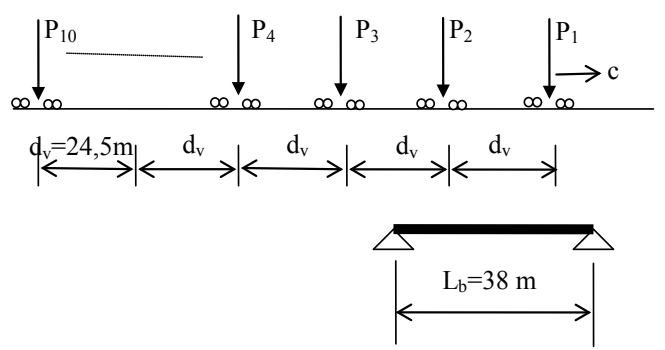

Fig. 3 The beam subjected to the series of loads $(P 1+P 2+\ldots . . P 10)$ moving across the beam

Input parameters: The bending stiffness of the bridge for the two rail loading model is:

$E I=7.58 .10^{7} \mathrm{kNm}^{2}$,

the beam mass per unit length $m_{1}=m_{1(\mathrm{BStr})}+m_{1(\text { Sup })}=3.18 \mathrm{t} / \mathrm{m}$,

$\omega_{(1)}=\frac{\pi^{2}}{L_{b}^{2}} \sqrt{\frac{E I}{\bar{m}_{1}}}=33.34\left[\mathrm{~s}^{-1}\right]$

the first circular frequency of the bridge $=33.34\left[\mathrm{~s}^{-1}\right]$, ${ }^{\left.(11) c_{2, r s}=65\right)} \alpha_{(1)}=\frac{\omega_{(1) d r}}{\omega_{(1)}}=\frac{\pi^{(1)} c_{2, r e s}}{L_{b} \omega_{1}}=0.1612$,

${ }^{\left.(1) c_{2, r s}\right)} \alpha_{(1)}=\frac{c}{c_{c r}}=\frac{65.03}{403.27}=0.1613$,

$c_{c r}=\frac{\pi}{L_{b}} \sqrt{\frac{E I}{m_{1}}}=403.27 \mathrm{~m} / \mathrm{s}$ is the critical speed,

$\hat{w}_{(1) s t}\left(L_{b} / 2\right)=\frac{2 P L_{b}^{3}}{\pi^{4} E I}=0.00791 \mathrm{~m}$ is the amplitude of the static deflection induced by vehicle, corresponding to the 1 -st mode of vibrations

${ }^{\left.(1) C_{2, r e s}\right)} \omega_{(1) d r}=\frac{\pi^{(1)} c_{2, \text { res }}}{L_{b}}=5.3735\left[\mathrm{~s}^{-1}\right]$,

$\frac{\hat{w}_{(1) s t}\left(L_{b} / 2\right)}{\left(1-{ }^{\left.(1)_{C, r s e}\right)} \alpha_{1}^{2}\right)}=\frac{0.0079}{1-0.1612^{2}}=0.0081 \mathrm{~m}$,

$\frac{L_{b}}{(1)_{2, n s}}=\frac{38}{65.03}=0.5843 \mathrm{~s}, \frac{d_{v}}{(1) c_{2, r e s}}=\frac{24.5}{65.03}=0.3767 \mathrm{~s}$,

the damping coefficient $=0.1327 \mathrm{~s}$.

\subsection{Components of the beam deflection due to the sequence of load IC-cars $\left(\boldsymbol{P}_{1}+\boldsymbol{P}_{2}+\ldots \boldsymbol{P}_{10}\right)$}

Components of the beam response due to the sequence of load IC-cars $\left(P_{1}+P_{2}+\ldots P_{10}\right)$ are defined by expressions (7).
- The quasi-static component ${ }^{\left(c_{2, \text { res }}=65\right)} \boldsymbol{w}_{(1) s t,\left(P_{1}, P_{2}, \ldots P_{10}\right)}\left(L_{b} / 2, t\right)$

The quasi-static component of the response includes the effect of forces moving direct over the beam (without damping) and is defined by the first term in Equation (7). Because of drawing the displacement $w$ in the downward direction in Equation (11), the minus sign is applied. The result is plotted in Fig. 4.

${ }^{\left(c_{2, \text { res }}=65\right)} \boldsymbol{W}_{(1) s t,\left(P_{1}, P_{2}, \ldots P_{10}\right)}\left(L_{b} / 2, t\right)=$

$=-\left[\frac{\hat{w}_{(1) s t}\left(L_{b} / 2\right)}{1-{ }^{(c)} \alpha_{(1)}^{2}} \sum_{n=0}^{M=9} \sin \left({ }^{\left(c_{2, \text { res }}\right)} \omega_{(1) d r}\left(t-\frac{n d_{v}}{c_{2, \text { res }}}\right)\right) H_{(n)}\right]$

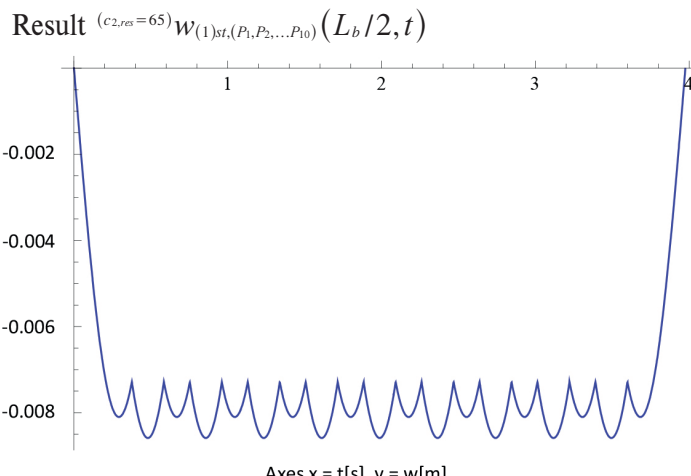

Axes $x=t[s], y=w[m]$
the load $\left(P_{1}, P_{2} \ldots P_{10}\right)=10 \times 528 \mathrm{kN}$ that move direct over the beam $\mathrm{t}=0$ to $3.97 \mathrm{~s}$

Fig. 4 The quasi-static component of the deflection

- The dynamic component ${ }^{\left(c_{2, r e s}=65\right)} \boldsymbol{w}_{(1) d y n,\left(P_{1}, P_{2}, \ldots P_{10}\right)}^{(1)}\left(L_{b} / 2, t\right)$

The dynamic component of the beam deflection includes the response defined by the second term in Equation (7). This dynamic response is plotted in Fig. 5.

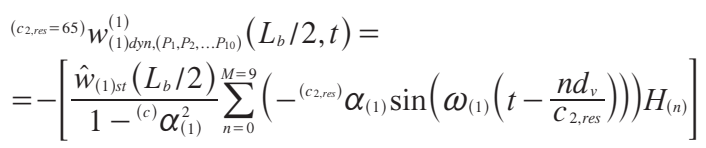

$\operatorname{Result}^{\left(c_{2, \text { res }}=65\right)} \boldsymbol{w}_{(1) d y n,\left(P_{1}, P_{2}, \ldots, P_{10}\right)}^{(1)}\left(L_{b} / 2, t\right)$

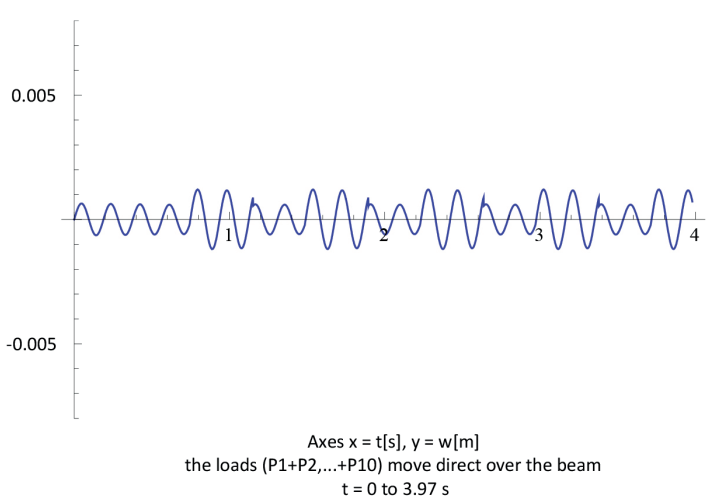

Fig. 5 The dynamic component 
- The total deflection beam response ${ }^{\left(c_{2,2, r s}=65\right)} w_{(1),\left(P_{1}, P_{2}, P_{10}\right)}^{\left(P_{10}\right.}\left(L_{b} / 2, t\right)$ corresponding to the load $\left(\boldsymbol{P}_{\boldsymbol{P}}, \boldsymbol{P}_{2}, \ldots \boldsymbol{P}_{10}\right)$ moving directly over the beam

The total deflection response ${ }^{\left(c_{2, r s}=65\right)} w_{(1),\left(P_{1}, P_{2}, \ldots, P_{10}\right)}^{\left({ }^{2}\right.}\left(L_{b} / 2, t\right)$ is superposition of the quasi-static and the dynamic component. The result is plotted in Fig. 6.
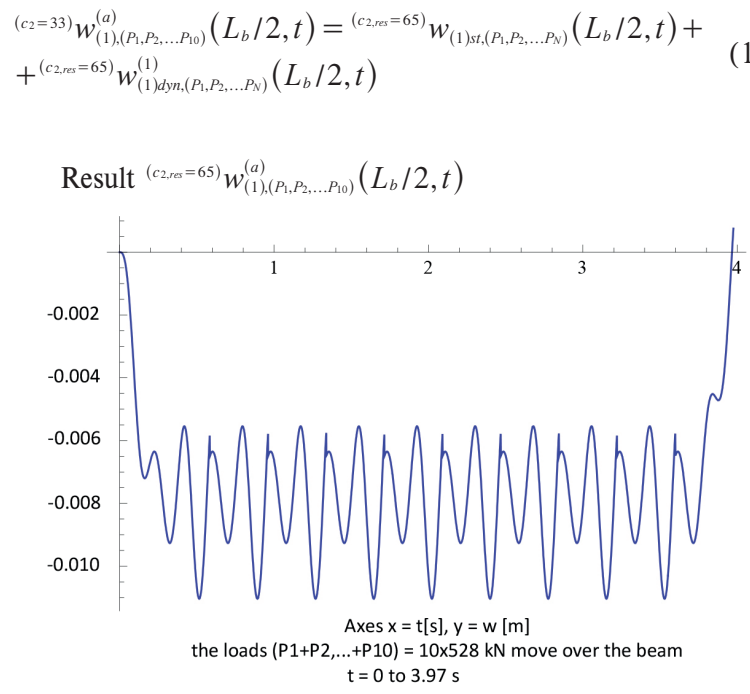

Fig. 6 The deflection response

The dynamic amplifier factor ${ }^{\left(c_{2}=33\right)} \Delta_{d y,\left(P_{1}, P_{2}, \ldots P_{10}\right)}^{(a)}=\frac{0.011}{0.0092}=1.1957$.

- The dynamic component ${ }^{\left(c_{2, r e s}=65\right)} \boldsymbol{w}_{(1) d y n,\left(P_{1}, P_{2}, \ldots, P_{10}\right)}^{(2)}\left(L_{b} / 2, t\right)$ for the load moving $\left(\boldsymbol{P}_{1}, \boldsymbol{P}_{2} \ldots . \boldsymbol{P}_{10}\right)$ out of the span

This dynamic component belongs to loads that have passed the beam, but they still influence the response. The result is plotted in, Fig. 7.

$$
\begin{aligned}
& { }^{\left(c_{2, r a s}=65\right)} \boldsymbol{w}_{(1) d y n,\left(P_{1}, P_{2}, \ldots P_{10}\right)}^{(2)}\left(L_{b} / 2, t\right)=- \\
& -\left[\begin{array}{l}
\frac{\hat{w}_{(1) s t}\left(L_{b} / 2\right)}{1-{ }^{\left(c_{2, r s s}\right)} \alpha_{(1)}^{2}} \\
\sum_{n=0}\left(-{ }^{\left(c_{2, r e s}\right)} \alpha_{(1)} e^{-\omega_{d}\left(t-\frac{n d_{v}}{c_{2}}\right)} \sin \omega_{(1)}\left(t-\frac{n d_{v}}{c_{2, \text { res }}}-\frac{L_{b}}{c_{2, \text { res }}}\right)\right) H_{(n)}
\end{array}\right]
\end{aligned}
$$

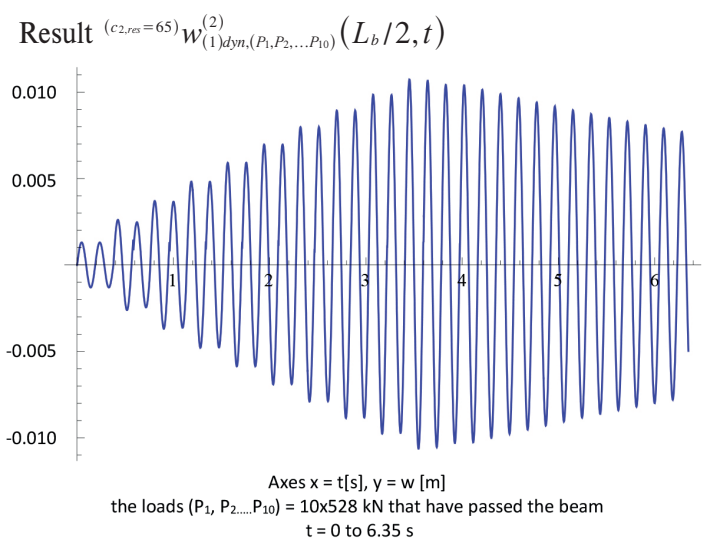

Fig. 7 The dynamic component

\section{- Components of the total response}

The components of the total deflection response $\left.{ }^{\left(c_{2}, \text { rgs }\right.}=65\right) w_{(1),\left(P_{1}, P_{2}, \ldots P_{10}\right)}\left(L_{b} / 2, t\right)$, belong to the load $\left(P_{p}, P_{2, \ldots .} P_{10}\right)$ that move directly over the beam and to the loads that have passed the beam are depicted in Fig. 8. The components are described by Equations (9), (10) and (12). The components

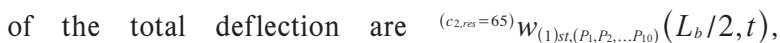
$\left\{{ }^{\left(c_{2, r s}=65\right)} \boldsymbol{w}_{(1) d y n,\left(P_{1}, P_{2}, \ldots, P_{10}\right)}^{(1)}\left(L_{b} / 2, t\right)+{ }^{\left(c_{2, r s s}=65\right)} \boldsymbol{w}_{(1) d y n,\left(P_{1}, P_{2}, \ldots P_{10}\right)}^{(2)}\left(L_{b} / 2, t\right)\right\}$

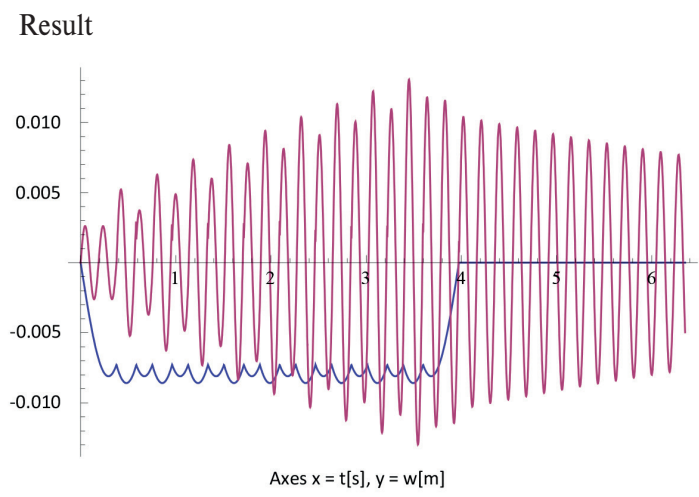

Fig. 8 Components of the total deflection:

\subsection{The total response due to the series of loads IC-cars $\left(\mathrm{P}_{1}+\mathbf{P}_{2}+\ldots \mathbf{P}_{10}\right)$}

The total response $\left.{ }^{\left(c_{2}, \text { res }\right.}=65\right) w_{(1),\left(P_{1}, P_{2}, \ldots P_{10}\right)}^{(a+2)}\left(L_{b} / 2, t\right)$ due to the series of load IC-cars $\left(P_{I}+P_{2}+\ldots P_{10}\right)$ is defined by superposition of the quasi-static and the dynamic components described by expressions (11), (12) and (14). The total beam response ${ }^{(c)} \boldsymbol{w}_{(1),\left(P_{1}, P_{2}, \ldots P_{N}\right)}^{(a+2)}\left(L_{b} / 2, t\right)$ can be symbolically written as the superposition of effects:

${ }^{\left(c_{2, r s}=65\right)} w_{(1),\left(P_{1}, P_{2}, \ldots, P_{10}\right)}^{(a+2)}\left(L_{b} / 2, t\right)={ }^{\left(c_{2, r e s}\right)} w_{(1) s t,\left(P_{1}, P_{2}, \ldots, P_{10}\right)}\left(L_{b} / 2, t\right)+$ $+{ }^{\left(c_{2, r e s}\right)} \boldsymbol{w}_{(1) d y,\left(P_{1}, P_{2}, \ldots, P_{10}\right)}^{(1)}\left(L_{b} / 2, t\right)+{ }^{\left(c_{2, r e s}\right)} \boldsymbol{w}_{(1) d y n_{n},\left(P_{1}, P_{2}, \ldots, P_{10}\right)}^{(2)}\left(L_{b} / 2, t\right)$

The result is plotted in Fig. 9.

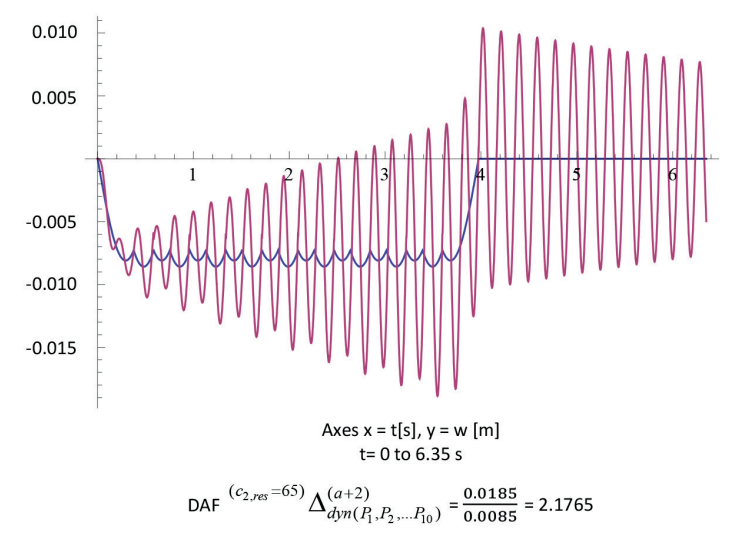

Fig. 9 The total beam displacement response 
Comparison of the dynamic amplifier factor ${ }^{\left(c_{2, n s}=33\right)} \Delta_{d y,\left(P_{1}, P_{2}, \ldots P_{10}\right)}^{(a)}=\frac{0.011}{0.0092}=1.1957$ for the deflection corresponding only the load acting directly on the beam and the factor ${ }^{\left(c_{2}, r s=65\right)} \Delta_{d y,\left(P_{1}, P_{2}, \ldots, P_{10}\right)}^{(a+2)}=\frac{0.0185}{0.0085}=2.1765$ involving also the effect of the residual free vibration of the beam caused by moving loads that have passed the beam, demonstrates the strong increase of the DAF at $54.93 \%$.

\section{Conclusions}

The dynamic response of bridges of small and medium spans, is markedly influenced just by composition of the periodic load of moving vehicles, due to the loading interval of vehicles, the rate of the moving loads, and the bridge length. The total dynamic deflections ${ }^{\left(c_{2, r a s}=33\right)} w_{(1),\left(P_{1}, P_{2}, \ldots P_{10}\right)}^{(a+2)}\left(L_{b} / 2, t\right)$ due to load by the series of IC-cars $\left(P_{1}+P_{2}+\ldots P_{N}\right)$ is defined by superposition of the quasistatic and the dynamic components described by expressions (11), (12) and (14). For small and medium spans and for low speeds $\mathrm{c}<160 \mathrm{~km} / \mathrm{h}$, the response does not lead to a significant increase, but at speeds $\mathrm{c} \geq 160 \mathrm{~km} / \mathrm{h}$ the dynamic amplifier factor ${ }^{\left(c_{2}=33\right)} \Delta_{d y n,\left(P_{1}, P_{2}, \ldots P_{N}\right)}^{(a n c r e a s e s ~ s i g n i f i c a n t l y ~ i n ~ p a r t i c u l a r ~ d u e ~ t o ~ t h e ~}$ dynamic components ${ }^{(c)} \boldsymbol{w}_{(1),\left(P_{1}, P_{2}, \ldots P_{N}\right)}^{(2)}\left(L_{b} / 2, t\right)$.

Comparison results for the train speed $c_{2}=33 \mathrm{~m} / \mathrm{s}$ and $c_{2, \text { res }}$ $=65 \mathrm{~m} / \mathrm{s}$ depicted on the Fig. 10 confirms the above statement.

The occurrence of resonant speeds $c_{i, \text { res }}$ results from parameters of the bridge and the train (distance axles of the train and the train speed). The resonant speeds $c_{\text {ires }}$ are reflected in a cumulative increase in dynamic response. With an increasing number of load forces $n=1,2, \ldots N$, for $M$-forces moving along the bridge and $K$-forces that have passed the beam, the deflection amplitude ${ }^{\left(c_{2, r a}=33\right)} \boldsymbol{w}_{\left.(1), P_{1}, P_{2}, \ldots, P_{10}\right)}^{(a)+2}\left(L_{b} / 2, t\right)$ increases.

\section{Acknowledgment}

This work was supported by the projects of Slovak Science Agency VEGA No.1/0336/15 and No. 2/0033/15 of the Slovak republic.

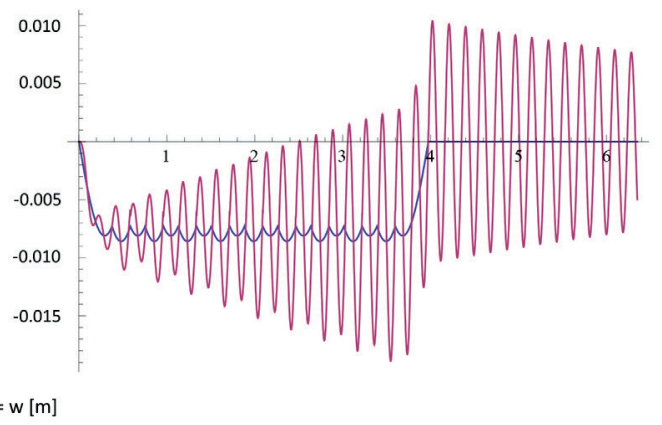

Fig. 10 Comparison the total dynamic response for the train speed $c_{2}=33 \mathrm{~m} / \mathrm{s}$ and $c_{2, r e s}=65 \mathrm{~m} / \mathrm{s}$

\section{References}

[1] KOLOUSEK, V.: Structural dynamic - Part I. (in Czech). SNTL, Praha, p. 263, 1954.

[2] BIGGS, J. M.: Structural Mechanics. McGraw-Hill, New York, 1964.

[3] FRYBA, L.: Vibration of Solids and Structures under Moving Loads. Telford, London, 1999.

[4] STN 1991-2: The European Standard EN 1991-2 (in Slovak), 2006.

[5] JANG, Y. B., LIN, C. W.: Vehicle-Bridge Interaction Dynamics and Potential Applications. Journal of Sound and Vibration, 284, 205-226, 2005.

[6] LI, J., SU, M.: The Resonant Vibration for a Simply Supported Girder Bridge under High-Speed Trains. Journal of Sound and Vibration, 224(5), 897-915, 1999.

[7] NAPRSTEK, J.: Resonance Speeds of Axle Forces Row Moving Along a Beam. Colloquium "Dynamics of Machines 2000”, Czech Republic, 2000.

[8] https://www.amazon.com/Wolfram-Mathematica 8.0.1 [online]. 